\title{
Verzeichnis der Autoren
}

Prof. Dr. Markus Achatz

Mitglied des Österreichischen Verfassungsgerichtshofes, Universitätsprofessor, Johannes Kepler Universität, Institut für Finanzrecht, Steuerrecht und Steuerpolitik, Steuerberater, Partner, leitner ${ }^{\text {leitner }}$, Linz

Prof. Dr. Heribert M. Anzinger

Universitätsprofessor, Universität Ulm, Institut für Rechnungswesen und Wirtschaftsprüfung

Dr. Stefanie Beinert

Rechtsanwältin, Steuerberaterin, Hengeler Mueller, Frankfurt a.M.

Jaap W. van den Berge

Raadsheer in buitengewone Dienst Hoge Raad, Den Haag

Walter Bode

Dipl.-Kfm., Richter am Bundesfinanzhof, München

Dr. Peter Brandis

Richter am Bundesfinanzhof, München

Prof. Jürgen Brandt

Richter am Bundesfinanzhof, Honorarprofessor, Bergische Universität Wuppertal

Dr. Stefan Breinersdorfer

Ministerialdirigent, Ministerium der Finanzen Rheinland-Pfalz, Mainz

Prof. Dr. Axel Cordewener, LL.M.

Universitätsprofessor, Katholieke Universiteit Leuven, Rechtsanwalt, Flick Gocke Schaumburg, Bonn

Prof. Dr. Marc Desens

Universitätsprofessor, Universität Leipzig, Lehrstuhl für Öffentliches Recht, insbes. Steuerrecht und Öffentliches Wirtschaftsrecht

Prof. Dr. Christian Dorenkamp, LL.M.

Deutsche Telekom AG, Honorarprofessor, Universität zu Köln 
Prof. Dr. Klaus-Dieter Drüen

Universitätsprofessor, Ludwig-Maximilians-Universität München, Lehrstuhl für Deutsches, Europäisches und Internationales Steuerrecht und Öffentliches Recht, Richter am Finanzgericht Düsseldorf

Prof. Dr. Tina Ehrke-Rabel

Universitätsprofessorin, Karl-Franzens-Universität Graz, Institut für Finanzrecht

Prof. Dr. Michael Eichberger

Richter des Bundesverfassungsgerichts a.D., Honorarprofessor, Eberhard Karls Universität Tübingen

Dr. Thomas Eisgruber

Ministerialrat, Bayerisches Staatsministerium der Finanzen, für Landesentwicklung und Heimat, München

Prof. Dr. Joachim Englisch

Universitätsprofessor, Westfälische Wilhelms-Universität Münster, Direktor des Instituts für Steuerrecht

Prof. Dr. Jutta Förster

Richterin am Bundesfinanzhof, Honorarprofessorin, Universität Osnabrück

Dr. Michael Geissler

Richter am Bundesfinanzhof, München

Dr. Stephan Geserich

Richter am Bundesfinanzhof, München

Prof. Dr. Dietmar Gosch

Vorsitzender Richter am Bundesfinanzhof a.D., Honorarprofessor, ChristianAlbrechts-Universität zu Kiel, Rechtsanwalt, Steuerberater, WTS Group, Hamburg

Dr. Christian Graw

Richter am Finanzgericht Düsseldorf

Prof. Dr. Joachim Hennrichs

Universitätsprofessor, Universität zu Köln, Lehrstuhl für Bürgerliches Recht,

Bilanz- und Steuerrecht

Prof. Dr. Andreas Herlinghaus

Richter am Bundesfinanzhof, Honorarprofessor, Universität Bonn 
Monika Hermanns

Richterin des Bundesverfassungsgerichts, Karlsruhe

Prof. Dr. Bernd Heuermann

Vorsitzender Richter am Bundesfinanzhof, Honorarprofessor, Ruprecht-Karls-

Universität Heidelberg

Prof. Dr. Johanna Hey

Universitätsprofessorin, Universität zu Köln, Direktorin des Instituts für Steuerrecht

Prof. Dr. Roland Ismer, MSc Econ. (LSE)

Universitätsprofessor, Friedrich-Alexander-Universität Erlangen-Nürnberg, Lehrstuhl für Steuerrecht und Öffentliches Recht

Prof. Dr. Monika Jachmann-Michel

Vorsitzende Richterin am Bundesfinanzhof, Honorarprofessorin, Ludwig-

Maximilians-Universität München

Prof. Dr. Harald Jatzke

Vorsitzender Richter am Bundesfinanzhof, Honorarprofessor, Eberhard Karls Universität Tübingen

Prof. Dr. Heike Jochum

Universitätsprofessorin, Universität Osnabrück, Institut für Finanz- und Steuerrecht

Prof. Dr. Christian Kaeser

Siemens AG, Honorarprofessor, Wirtschaftsuniversität Wien

Prof. Dr. Simon Kempny, LL.M.

Universitätsprofessor, Universität Bielefeld, Lehrstuhl für Öffentliches Recht und Steuerrecht

Prof. Dr. Ferdinand Kirchhof

Vizepräsident des Bundesverfassungsgerichts, Universitätsprofessor (em.), Eberhard Karls Universität Tübingen

Prof. Dr. Gregor Kirchhof, LL.M.

Universitätsprofessor, Universität Augsburg, Direktor des Instituts für Wirtschaftsund Steuerrecht, Lehrstuhl für Öffentliches Recht, Finanzrecht und Steuerrecht 
Prof. Dr. Dres. h.c. Paul Kirchhof

Richter des Bundesverfassungsgerichts a.D., Seniorprofessor distinctus für Staats- und Steuerrecht, Ruprechts-Karls-Universität Heidelberg, Institut für Finanz- und Steuerrecht

Prof. DDr. Georg W. Kofler, LL.M.

Universitätsprofessor, Johannes Kepler Universität Linz, Institut für Finanzrecht, Steuerrecht und Steuerpolitik

Prof. Dr. Juliane Kokott, LL.M., S.J.D.

Universitätsprofessorin, Generalanwältin am Gerichtshof der Europäischen

Union, Luxemburg

Dr. Robert Jan Koopmann

Vizepräsident des Hoge Raad, Den Haag

Martin Kreienbaum

Ministerialdirigent, Bundesministerium der Finanzen, Berlin

Dr. Roland Krüger

Richter am Bundesfinanzhof, München

Ulrich Krüger

Vorsitzender Richter am Bundesfinanzhof, München

Prof. Dr. Marcel Krumm

Universitätsprofessor, Westfälische Wilhelms-Universität Münster, Lehrstuhl für

Öffentliches Recht und Steuerrecht, Richter am Finanzgericht Münster

Prof. Dr. Hanno Kube, LL.M.

Universitätsprofessor, Ruprecht-Karls-Universität Heidelberg, Direktor des

Instituts für Finanz- und Steuerrecht

Dr. Egmont Kulosa

Richter am Bundesfinanzhof, München

Prof. Dr. DDr. h.c. Michael Lang

Universitätsprofessor, Wirtschaftsuniversität Wien, Institut für Österreichisches und Internationales Steuerrecht

Prof. Dr. Hans-Friedrich Lange

Vorsitzender Richter am Bundesfinanzhof a.D., Honorarprofessor, Westfälische Wilhelms-Universität Münster 
Prof. Dr. Moris Lehner

Universitätsprofessor (em.), Ludwig-Maximilians-Universität München, Lehrstuhl für Öffentliches Wirtschafts- und Steuerrecht, Leiter der Forschungstelle für Europäisches und Internationales Steuerrecht

Dr. Christian Levedag

Richter am Bundesfinanzhof, München

Prof. Dr. Matthias Loose

Richter am Bundesfinanzhof, Honorarprofessor, Ruhr-Universität Bochum

Prof. Dr. Jürgen Lüdicke

Universitätsprofessor, Universität Hamburg, Lehrstuhl für Internationales

Steuerrecht, Rechtsanwalt, Steuerberater, PricewaterhouseCoopers, Hamburg

Dr. Markus Märtens

Richter am Bundesfinanzhof, München

Prof. Dr. h.c. Rudolf Mellinghoff

Präsident des Bundesfinanzhofs, Richter des Bundesverfassungsgerichts a.D., Honorarprofessor, Eberhard Karls Universität Tübingen

Prof. Dr. André Meyer

Universitätsprofessor, Universität Bayreuth, Lehrstuhl für Bürgerliches Recht und Steuerrecht, Gesellschafts- und Bilanzrecht

Dr. Rolf Möhlenbrock

Ministerialdirigent, Bundesministerium der Finanzen, Berlin

Prof. Dr. Andreas Musil

Universitätsprofessor, Universität Potsdam, Lehrstuhl für Öffentliches Recht, insbesondere Verwaltungs- und Steuerrecht

Dr. Eva Oertel

Oberregierungsrätin, Bayerisches Staatsministerium der Finanzen, für Landesentwicklung und Heimat, München

Prof. Dr. Ulrich Eugen Palm

Universitätsprofessor, Universität Hohenheim, Lehrstuhl für Öffentliches Recht, Finanz- und Steuerrecht

Dr. Volker Pfirrmann

Richter am Bundesfinanzhof, München 
Prof. Dr. Henning Radtke

Richter des Bundesverfassungsgerichts, Honorarprofessor, Universität Hannover

Dr. Eckart Ratschow

Richter am Bundesfinanzhof, München

Prof. Dr. Ekkehart Reimer

Universitätsprofessor, Ruprecht-Karls-Universität Heidelberg, Direktor des Instituts für Finanz- und Steuerrecht

Prof. Dr. Dr. h.c. Klaus Rennert

Präsident des Bundesverwaltungsgerichts, Honorarprofessor, Universität Freiburg

Prof. Dr. Thomas Rödder

Wirtschaftsprüfer, Steuerberater, Flick Gocke Schaumburg, Honorarprofessor, Universität zu Köln

Prof. Dr. Alexander Rust, LL.M.

Universitätsprofessor, Wirtschaftsuniversität Wien, Institut für Österreichisches und Internationales Steuerrecht

Dr. Ulrich Schallmoser

Richter am Bundesfinanzhof, München

Prof. Dr. Deborah Schanz

Universitätsprofessorin, Ludwig-Maximilians-Universität München, Vorstand des Instituts für Betriebswirtschaftliche Steuerlehre

Prof. Dr. Rainer Schlegel

Präsident des Bundessozialgerichts, Honorarprofessor, Justus-Liebig-Universität Gießen

Eckehard Schmidt

Ministerialdirigent a.D., Bayerisches Staatsministerium der Finanzen, für

Landesentwicklung und Heimat, München

Ingrid Schmidt

Präsidentin des Bundesarbeitsgerichts, Erfurt

Prof. Dr. Michael Schmitt

Ministerialdirigent a.D., Ministerium für Finanzen Baden-Württemberg, Honorarprofessor, Universität Mannheim 
Prof. Dr. Stefan Schneider

Vorsitzender Richter am Bundesfinanzhof, Honorarprofessor, Universität Mannheim

Prof. Dr. Dr. h.c. Wolfgang Schön

Honorarprofessor, Ludwig-Maximilians-Universität, Direktor am Max-PlanckInstitut für Steuerrecht und Öffentliche Finanzen, München

Prof. Dr. Jens Schönfeld

Rechtsanwalt, Fachanwalt für Steuerrecht, Diplom-Kaufmann, Partner, Flick Gocke Schaumburg, Honorarprofessor, Universität Osnabrück

Dr. Michael Schwenke

Richter am Bundesfinanzhof, München

Prof. Dr. Roman Seer

Universitätsprofessor, Ruhr-Universität Bochum, Lehrstuhl für Steuerrecht

Prof. Dr. Christian Seiler

Universitätsprofessor, Eberhard Karls Universität Tübingen, Lehrstuhl für Staats- und Verwaltungsrecht, Finanz- und Steuerrecht, Mitglied des Verfassungsgerichtshofs Baden-Württemberg

Michael Sell

Ministerialdirektor, Bundesministerium der Finanzen, Berlin

Prof. Dr. Susanne Sieker

Universitätsprofessorin, Martin-Luther-Universität Halle-Wittenberg, Lehrstuhl für Bürgerliches Recht, Handelsrecht, Steuerrecht und Wirtschaftsrecht

Prof. Dr. Madeleine Simonek

Universitätsprofessorin, Universität Zürich, Lehrstuhl für Schweizerisches und Internationales Steuerrecht

Thomas Stadelmann

Dipl. Steuerexperte, Richter am Schweizerischen Bundesgericht, Lausanne

Prof. Dr. Thomas Stapperfend

Präsident des Finanzgerichts Berlin-Brandenburg, Honorarprofessor, Humboldt-Universität zu Berlin

Prof. Dr. Claus Staringer

Universitätsprofessor, Wirtschaftsuniversität Wien, Institut für Österreichisches und Internationales Steuerrecht 
Dr. Franz Philipp Sutter

Richter am Österreichischen Verwaltungsgerichtshof, Wien

Prof. Dr. Christian Thiemann

Universitätsprofessor, Johannes Gutenberg-Universität Mainz, Lehrstuhl für

Öffentliches Recht, Europarecht, Finanz- und Steuerrecht

Prof. Dr. Andreas Thier

Universitätsprofessor, Universität Zürich, Lehrstuhl für Rechtsgeschichte, Kirchenrecht, Rechtstheorie und Privatrecht

Dr. Nils Trossen

Richter am Bundesfinanzhof, München

Hermann-Ulrich Viskorf

Vizepräsident des Bundesfinanzhofs a.D., Bad Aibling

Prof. Dr. Roland Wacker

Vorsitzender Richter am Bundesfinanzhof, Honorarprofessor, Universität Hohenheim

Dr. Christoph Wäger

Richter am Bundesfinanzhof, München

Prof. Dr. Christian Waldhoff

Universitätsprofessor, Humboldt-Universität zu Berlin, Lehrstuhl für Öffentliches Recht und Finanzrecht

Michael Wendt

Vorsitzender Richter am Bundesfinanzhof, München

Prof. Dr. Rainer Wernsmann

Universitätsprofessor, Universität Passau, Lehrstuhl für Staats- und Verwaltungsrecht, insbesondere Finanz- und Steuerrecht

Prof. Dr. Franceska Werth

Richterin am Bundesfinanzhof, Honorarprofessorin, Albert-Ludwigs-Universität Freiburg

Prof. Dr. Nikolaus Zorn

Senatspräsident am Österreichischen Verwaltungsgerichtshof, Universitätsprofessor, Universität Innsbruck, Institut für Unternehmens- und Steuerrecht 